OPEN ACCESS

Edited by:

Mike Taylor,

University of Auckland, New Zealand

Reviewed by:

David William Waite

The University of Queensland,

Australia

Charles K. Lee,

University of Waikato, New Zealand

*Correspondence: Hirokazu Tsuji

hirokazu-tsuji@yakult.co.jp

${ }^{\dagger}$ Present Address: Koji Nomoto,

Division of Molecular Microbiology, Tokyo University of Agriculture, Tokyo,

Japan

Specialty section:

This article was submitted to

Microbial Symbioses,

a section of the journal

Frontiers in Microbiology

Received: 01 August 2017 Accepted: 08 June 2018

Published: 29 June 2018

Citation:

Tsuji H, Matsuda K and Nomoto K (2018) Counting the Countless:

Bacterial Quantification by Targeting rRNA Molecules to Explore the Human Gut Microbiota in Health and Disease.

Front. Microbiol. 9:1417.

doi: 10.3389/fmicb.2018.01417

\section{Counting the Countless: Bacterial Quantification by Targeting rRNA Molecules to Explore the Human Gut Microbiota in Health and Disease}

\author{
Hirokazu Tsuji ${ }^{1 *}$, Kazunori Matsuda ${ }^{2}$ and Koji Nomoto ${ }^{1+}$ \\ ${ }^{1}$ Basic Research Department, Yakult Central Institute, Tokyo, Japan, ${ }^{2}$ Yakult Honsha European Research Center for \\ Microbiology ESV, Gent-Zwijnaarde, Belgium
}

Over the past decade, the advent of next-generation-sequencing tools has revolutionized our approach to understanding the human gut microbiota. However, numerical data on the gut bacterial groups - particularly low-cell-count microbiota, such as indigenous pathobionts, that are otherwise important components of the microbiota-are relatively limited and disparate. As a result, the comprehensive quantitative structure of the human gut microbiota still needs to be fully defined and standardized. With the aim of filling this knowledge gap, we have established a highly sensitive quantitative analytical system that is based on reverse transcription-quantitative PCR and targets microbial rRNA molecules. The system has already been validated in the precise, sensitive, and absolute quantification of more than 70 target bacterial groups belonging to various human gut bacterial clades, including predominant obligate and facultative anaerobes. The system demonstrates sensitivity several hundred times greater than that of other rRNA-gene-targeting methods. It is thus an efficient and valuable tool for exhaustive analysis of gut microbiota over a wide dynamic range. Using this system, we have to date quantified the gut microbiota of about 2,000 healthy Japanese subjects ranging in age from 1 day to over 80 years. By integrating and analyzing this large database, we came across several novel and interesting features of the gut microbiota, which we discuss here. For instance, we demonstrated for the first time that the fecal counts of not only the predominant bacterial groups but also those at lower cell counts conform to a logarithmically normal distribution. In addition, we revealed several interesting quantitative differences in the gut microbiota of people from different age groups and countries and with different diseases. Because of its high analytic sensitivity, the system has also been applied successfully to other body niches, such as in characterizing the vaginal microbiota, detecting septicemia, and monitoring bacterial translocation. Here, we present a quantitative perspective on the human gut microbiota and review some of the novel microbial insights revealed by employing this promising analytical approach.

Keywords: age-related bacterial community, bacterial quantification, disease-specific dysbiosis, gut microbiota, RT-qPCR, YIF-SCAN 


\section{INTRODUCTION}

The human intestine harbors a highly complex microbial ecosystem (Turnbaugh et al., 2007) comprising as many as 1,000 different kinds of bacteria, collectively known as the intestinal microbiota, which plays a fundamental role in human health (Turnbaugh et al., 2006; Round and Mazmanian, 2009; Arumugam et al., 2011; Cho and Blaser, 2012; Yatsunenko et al., 2012; Koeth et al., 2013; Yoshimoto et al., 2013). The intestinal microbiota consists of various microbial communities that are present at different microbial cell counts and can be categorized as (a) predominant strictly anaerobic bacterial groups such as Clostridium coccoides group, Clostridium leptum subgroup, Bacteroides fragilis group and Bifidobacterium, (b) low-cellcount microbiota (LCCM) including facultative anaerobic groups such as Enterobacteriaceae, Lactobacillus, Enterococcus, and Staphylococcus, and (c) low-population-level indigenous pathobionts such as Clostridium difficile and Clostridium perfringens at various population densities (Ozaki et al., 2004; Tonooka et al., 2005; Lakshminarayanan et al., 2013). The cell counts of these diverse bacterial groups are highly controlled by "colonization resistance," which is a means by which the microbiota and the host maintain a stable balance (van der Waaij et al., 1971; Buffie and Pamer, 2013; Lawley et al., 2013).

Under normal conditions, the healthy intestinal microbiota exists in homeostasis with the host, whereby the population growth (and toxigenicity) of LCCM including pathobionts remains controlled. However, in a state of dysbiosis (i.e., intestinal microbial imbalance), these LCCM can multiply and cause serious problems in clinical settings (Gorkiewicz, 2009; Litvak et al., 2017; Samarkos et al., 2018). Therefore, to comprehensively assess the intestinal microbiota-a diverse microbial ecosystem with a wide dynamic range-it is essential that we precisely understand not only the relative abundance, but also the microbial cell counts of the predominant bacterial groups and the LCCM-including facultative anaerobes and indigenous pathobionts. Accordingly, even in this golden age of advanced sequencing tools, quantitative data corresponding to the "gold standard" viable bacterial count remain critically important.

Recently, Vandeputte et al. (2017) constructed a workflow for the quantitative microbiome profiling of fecal bacteria combining 16S rRNA gene PCR amplicon sequencing and flow cytometric quantification of microbial cells, and demonstrated that the microbial loads of healthy individuals can vary across a 10-fold dynamic range and also that microbial loads are a key driver for microbiota alterations in patients with Crohn's disease. The authors stated: "To enable genuine characterization of host-microbiota interactions, microbiome research must exchange ratios for counts" (Vandeputte et al., 2017). To address this, we have developed a sensitive and absolute quantitative analytical system (known in-house as Yakult-Intestinal-FloraScan; hereafter referred to as YIF-SCAN) that is based on reverse transcription-quantitative PCR (RT-qPCR) and targets rRNA molecules abundant in microbes (Matsuda et al., 2007, 2009). YIF-SCAN quantifies the predominant human intestinal bacteria at a sensitivity equivalent to that of qPCR or fluorescent in-situ hybridization (FISH) methods (detection limit: $10^{5}$ to $10^{8}$ cells/g feces); LCCM can be enumerated at sensitivities comparable to those of culture methods (detection limit: $10^{2}$ to $10^{3}$ cells/g feces: $\sim 100$ to 1,000 times greater than that of qPCR and 100,000 to 1,000,000 times greater than that of FISH) (Matsuda et al., 2007, 2009, 2012; Kubota et al., 2010; Kurakawa et al., 2012, 2013).

Using this analytical system, we have to date analyzed and reported the quantitative gut microbial composition of more than 1,900 healthy Japanese subjects ranging in age from 1 day to over 80 years (Nomoto et al., 2015). Recently, this database was further augmented by the addition of 451 healthy subjects (Hasegawa et al., 2015; Morita et al., 2015; Nakayama et al., 2015; Aizawa et al., 2016; Suzuki et al., 2017) and analyzed using several newly validated bacterial assays that were not previously executed. Taking into account the hypotheses and preliminary data from our previous investigations, we subjected this database to additional exhaustive statistical analyses that revealed some intriguing features of the gut microbiota. For instance, we demonstrate that fecal counts of not only the predominant obligate anaerobes but also the facultative anaerobes follow a logarithmic normal distribution. Prevotella, however, emerges as a surprising exception: it exhibits a multimodal distribution pattern. Interestingly, several age-dependent, gender-specific differences in intestinal microbial composition are also revealed by this intensive analysis. Furthermore, by integrating this database and using it as a reference, we reveal some important features of gut dysbiosis in several diseases and demonstrate regional differences in the intestinal microbiota among several Asian or Oceanic populations. YIF-SCAN analysis has also enabled us to precisely monitor the gut microbial changes that occur following the ingestion of probiotics or synbiotics. Given that quantitative data are likely to prove invaluable for understanding the roles of different gut microbes in health and disease, the high sensitivity of YIF-SCAN makes it a very useful tool in both gut microbiota exploration and microbial diagnosis in clinical practice.

\section{TARGETING rRNA MOLECULES FOR BACTERIAL QUANTIFICATION: A NEW FRONTIER IN GUT MICROBIOTA ANALYSIS}

As mentioned above, YIF-SCAN is based on RT-qPCR. Generally, bacteria contain thousands to tens of thousands of ribosomes, which include rRNA molecules that can be classified into 5S, 16S, and 23S subunits according to their sedimentation rate. However, only a few to a dozen copies of rRNA genes are encoded on the bacterial chromosome. Although FISH has been used to quantify bacteria by targeting rRNA molecules, the detection sensitivity of this method is only at the level of $10^{8}$ cells/g feces (Matsuki et al., 2004a). We speculated that quantitative analysis targeting rRNA molecules would greatly improve analytical sensitivity. When primers targeting the same rRNA gene sequence are used, there is no difference in the slope of the standard curve between qPCR and RT-qPCR, but the difference in the $y$-intercept (threshold cycle [Ct] value) reveals at least 100 times increased sensitivity (Matsuda et al., 2007, 2012; 
Kubota et al., 2010; Kurakawa et al., 2012; Ogata et al., 2015). Furthermore, rRNA-gene-based PCR often measures not only viable bacteria but also free bacterial DNA or the DNA from dead bacteria, whereas the detection of rRNA molecules by RT-qPCR is more closely associated with bacterial viability. In fact, studies using in vitro cultures have shown that, for several different bacterial strains, YIF-SCAN data corresponds to that obtained by the culture method throughout the growth phases and during the process of cell death (Matsuda et al., 2007; Kurakawa et al., 2012). RT-qPCR quantification of rRNA molecules thereby provides a combination of both cell abundance and viability, and is therefore an efficient and preferable method for quantifying live intestinal bacteria.

It should be noted that certain bacteria may remain underrepresented or underestimated in DNA-sequencing-based datasets because of technical anomalies such as a bias of PCR primers against particular 16S rRNA gene sequences or the use of DNA extraction methods that are not equally effective for all gut inhabitants (e.g., high vs. low $\mathrm{G}+\mathrm{C}$, or Gram positive vs. negative). Also, although sequencing methods generate tens of thousands of rRNA gene sequences per DNA sample, there could be several hundred operational taxonomic units in a given sample and hence it is possible that taxa with very low abundance could be overlooked (Tannock et al., 2016). Moreover, given that only a fragment of the rRNA gene is sequenced, the resolution may not be adequate and consistent enough for species-level discrimination. Given that it is challenging to design a universal primer that amplifies the gene sequences of all gut microbes with equal efficiency, to achieve detailed information it is necessary to enumerate bacterial groups by using specific as well as sensitive primer sets. To this end, YIF-SCAN uses thoroughly validated specific primers sets and targets $16 \mathrm{~S}$ rRNA molecules, which are present at huge copy numbers per cell (compared with gene copy numbers), thereby yielding high analytical sensitivity and precise and absolute quantification data (Matsuda et al., 2007, 2009). The YIF-SCAN analysis consists of the following operations: (1) collection and storage of feces, (2) isolation of fecal RNA, (3) preparation of reaction microplate for PCR assay, (4) RT-qPCR, and (5) calculation of bacterial count from PCR data. In order to automate the series of the operations of No. 2, 3, and 4, we have installed the following hardware: a liquid handling workstation, a robotic centrifuge, a whole rack tube capper/decapper, and a robotic handling machine. These hardware are connected with real-time PCR devices via the robotic handling machine, and the integrated system is automatically managed by the respective drivers and a scheduling software. In this system, the fecal samples are dispensed into 96 whole rack tubes and are controlled using barcode technology. The automation system has dramatically improved the throughput and reproducibility of YIF-SCAN, and has decreased the risks that researchers might be contaminated by fecal bacteria and chemicals during the RNA extracting operations.

For YIF-SCAN analysis, we established a pool of more than 70 primer sets and the corresponding nucleic acids (RNA) from standard strains of various gut microbiota (Table 1). A strain representing a target bacterial group was used as the standard strain. RNA was extracted from each standard strain by using the same method as for fecal samples in order to delete experimental biases associated with differences in amplification efficiency among primer sets and differences in RNA extraction efficiency among bacterial species. Using a pool of primer sets facilitated the quantification of a wide range of subgroups or species belonging to various human gut bacterial clades, including predominant obligate anaerobes, facultative anaerobes (as well as LCCM), and opportunistic or potential pathogens (Table 1). Notably, all of the primer sets had been thoroughly validated for specific, sensitive, and absolute quantification of the corresponding targets in human feces. In addition to the major predominant obligate anaerobes, this in-house validated pool encompassed facultative anaerobes present as LCCM in the intestine, thereby enabling an exhaustive analysis of gut microbiota across a wide dynamic range. For example, in the case of Lactobacillus, eight subgroups and species can be analyzed, whereas in the case of Bifidobacterium eight bacterial species commonly detected in humans can be examined. Detection systems for species that belong to the Gram-positive catalase-negative cocci, such as Streptococcus, Enterococcus, and Lactococcus which are prevalent in the human intestine have also been developed (Kubota et al., 2010). Furthermore, we also analyzed intestinal pathogens such as Vibrio cholerae/mimicus, Vibrio parahaemolyticus, and Campylobacter (Kurakawa et al., 2012; Nair et al., 2012), as well as several other opportunistic pathogens and fungi (Matsuda et al., 2012; Nagpal et al., 2015; Ogata et al., 2015).

In subsequent sections, we demonstrate some interesting features of the human gut microbiota that have been revealed with the use of this quantitative analytical system. We also introduce the potential application of the system in the clinical diagnosis of bacteremia.

\section{QUANTITATIVE DYNAMICS OF THE HUMAN GUT MICROBIOTA: INSIGHTS FROM MICROBIOTA ANALYSIS IN HEALTHY JAPANESE POPULATIONS}

The last decade has seen remarkable advances in elucidating the intimate relationship between the intestinal microbiota and aging (Hopkins et al., 2001; Woodmansey, 2007; Enck et al., 2009; Biagi et al., 2010; Tiihonen et al., 2010; Claesson et al., 2012; O’Toole, 2012; Yatsunenko et al., 2012; Rampelli et al., 2013; Odamaki et al., 2016). YIF-SCAN can quantify gut bacteria with reproducibility, thereby enabling us to easily and precisely integrate the data from many independent clinical studies and scrutinize the age-related dynamics of the microbiota. We used an integrated database of 11 major human intestinal bacterial genera, and species that have been quantified in different studies in a total of 1951 healthy Japanese subjects (age range: 1 day to 102 years; Supplementary Table 1) and used YIF-SCAN to provide a quantitative profiling of the major human gut bacterial groups of the gut microbiota in the context of host age (Matsuda et al., 2009; Bian et al., 2011; Nagata et al., 2011, 2016; Tsuji et al., 2012, 2014; Ohigashi et al., 2013; Aoki et al., 2014; Sato et al., 2014; Hasegawa et al., 2015; Morita et al., 2015; Nakayama et al., 2015; Wang et al., 2015; Aizawa et al., 2016). 
TABLE 1 | List of bacterial groups included in the YIF-SCAN analytical system.

\begin{tabular}{cc}
\hline Bacterial groups & $\begin{array}{c}\text { Detection limit } \\
\left(\log _{10} \text { cells/g }\right. \\
\text { feces })\end{array}$
\end{tabular}

\section{OBLIGATE ANAEROBES}

Clostridium coccoides group

Clostridium hathewayi

Clostridium indolis subgroup

Clostridium symbiosum

Fusicatenibacter saccharivorans

Genus Blautia

Clostridium nexile

Ruminococcus gnavus

Anaerostipes caccae

Ruminococcus lactaris

Eubacterium ramulus

Eubacterium rectale

Eubacterium eligens

Eubacterium ventriosum

Eubacterium hallii

Clostridium asparagiforme

Coprococcus comes

Coprococcus eutactus

Roseburia intestinalis

Clostridium hylemonae

Clostridium scindens

Genus Dorea

Ruminococcus torques

Clostridium leptum subgroup

Bacteroides fragilis group

Genus Bifidobacterium

Bifidobacterium adolescentis

Bifidobacterium angulatum

Bifidobacterium bifidum

Bifidobacterium breve

Bifidobacterium catenulatum group

Bifidobacterium dentium

Bifidobacterium longum subsp. longum

Bifidobacterium longum subsp. infantis

Atopobium cluster

Genus Prevotella

Clostridium perfringens

Clostridium difficile

\section{FACULTATIVE ANAEROBES}

Family Enterobacteriaceae

Escherichia coli

Genus Lactobacillus

Lactobacillus casei subgroup

Lactobacillus gasseri subgroup
Matsuki et al., 2002

Kurakawa et al.,

$2015 a$

4

3

4

3

4

3

3

4

3

3

3

2

3

3

3

4

4

4

2

Matsuki, 2007

Matsuki et al., 1998 2015b

Matsuki et al., 2004a

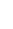

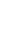

3

3

Kurakawa et al., 2015b

Matsuki et al., 2004b

Matsuki et al., 2002

Matsuda et al., 2009

Matsuda et al., 2012

Matsuda et al., 2007

Kurakawa et al., 2013

Matsuda et al., 2009

(Continued)
Kurakawa et al.,
TABLE 1 | Continued

\begin{tabular}{|c|c|c|}
\hline Bacterial groups & $\begin{array}{l}\text { Detection limit } \\
\text { ( } \log _{10} \text { cells/g } \\
\text { feces) }\end{array}$ & $\begin{array}{l}\text { References for } \\
\text { primer sets }\end{array}$ \\
\hline Lactobacillus plantarum subgroup & 3 & \\
\hline Lactobacillus reuteri subgroup & 3 & \\
\hline Lactobacillus ruminis subgroup & 3 & \\
\hline Lactobacillus sakei subgroup & 3 & \\
\hline Lactobacillus brevis & 3 & \\
\hline Lactobacillus fermentum & 5 & \\
\hline Lactobacillus fructivorans & 3 & \\
\hline Genus Enterococcus & 3 & \\
\hline Enterococcus faecalis & 3 & Kubota et al., 2010 \\
\hline Enterococcus caccae & 3 & \\
\hline Enterococcus cecorum & 4 & \\
\hline Enterococcus sulfureus subgroup & 4 & \\
\hline Enterococcus casseliflavus subgroup & 3 & \\
\hline Enterococcus avium subgroup & 3 & \\
\hline Enterococcus dispar & 3 & \\
\hline Enterococcus faecium subgroup & 4 & \\
\hline Enterococcus faecium & 4 & \\
\hline Genus Streptococcus & 4 & $\begin{array}{l}\text { Sakaguchi et al., } \\
2010\end{array}$ \\
\hline Streptococcus agalactiae & 4 & \\
\hline Streptococcus pyogenes & 4 & \\
\hline Streptococcus pneumonia/ mitis & 4 & \\
\hline Streptococcus salivarius/ thermophiles & 4 & Kubota et al., 2010 \\
\hline Lactococcus lactis subgroup & 4 & \\
\hline Lactococcus piscium subgroup & 4 & \\
\hline Genus Staphylococcus & 3 & Matsuda et al., 2009 \\
\hline \multicolumn{3}{|l|}{ PATHOGENS } \\
\hline Genus Pseudomonas & 4 & Matsuda et al., 2007 \\
\hline Vibrio cholerae/mimicus & 3 & $\begin{array}{l}\text { Kurakawa et al., } \\
2012\end{array}$ \\
\hline Vibrio parahaemolyticus/alginolyticus & 3 & \\
\hline Campylobacter jejuni/coli & 3 & \\
\hline Candida group & 2 & Ogata et al., 2015 \\
\hline Candida albicans & 3 & \\
\hline Candida glabrata & 2 & \\
\hline Candida krusei & 2 & \\
\hline Candida tropicalis & 2 & \\
\hline Candida parapsilosis & 2 & \\
\hline
\end{tabular}

Recent sequence analyses using bacterial 16S rRNA genes have reported that gut microbiota diversity in newborns undergoes a gradual increase early in life, but reaches a plateau by 3 years of age, at which time the microbial configuration and diversity become equivalent to those in adults (Nakayama, 2010; Yatsunenko et al., 2012; Odamaki et al., 2016). Further agerelated quantitative changes in intestinal bacterial populations have been shown by YIF-SCAN analysis the predominant bacterial populations and those present at lower levels, such as Enterobacteriaceae, lactobacilli, and pathobionts. Moreover, the data from our integrated database, namely bacterial counts 

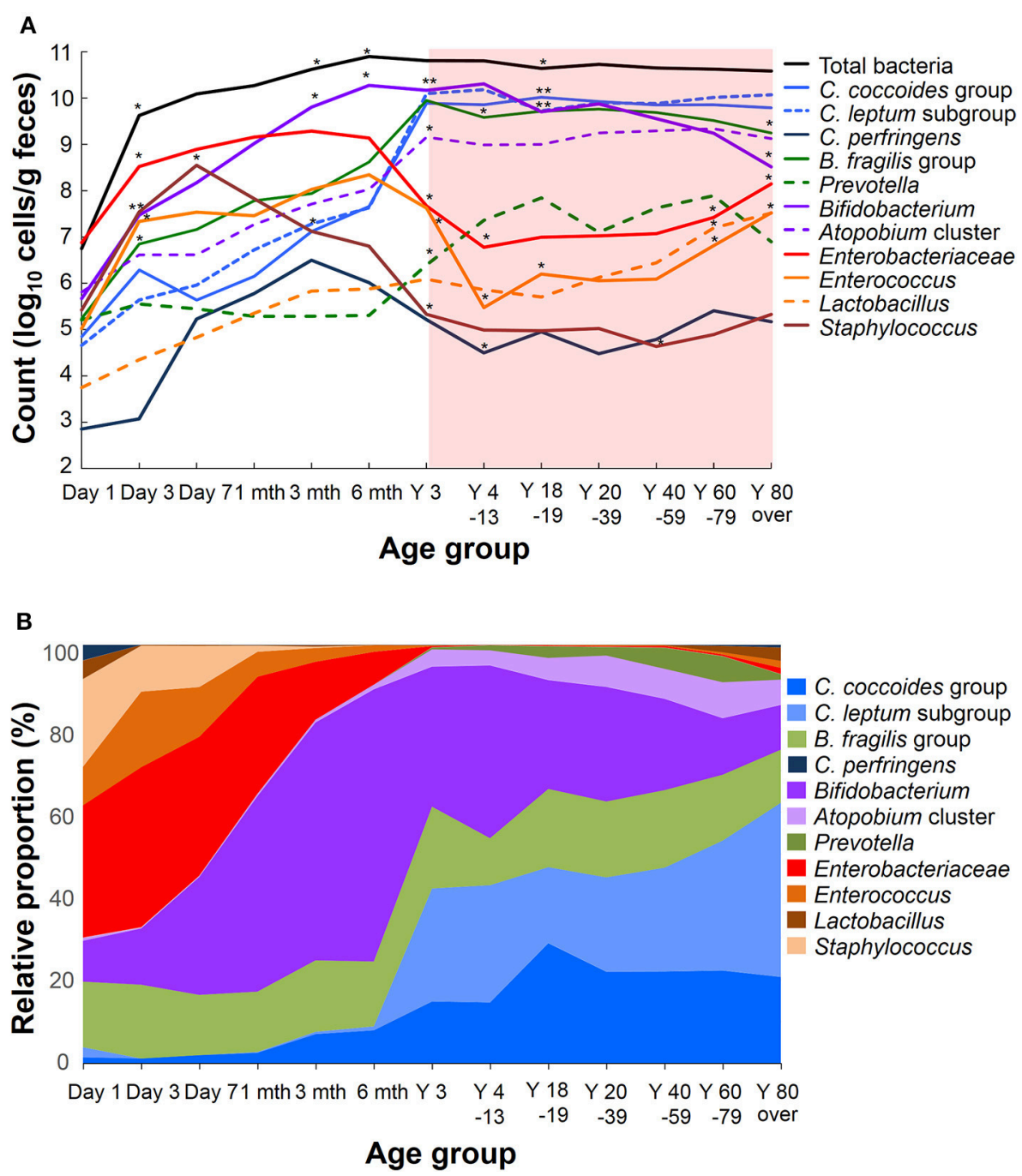

FIGURE 1 | Age-related differences in average counts $\mathbf{( A )}$ and relative proportions $\mathbf{( B )}$ of intestinal bacteria in healthy Japanese volunteers $(n=1951)$. An asterisk shows the significant difference between the count of a period and the preceding one $\left(\mathbf{A}\right.$, Steel-Dwass multiple comparison test, ${ }^{\star} P<0.05$, $\left.{ }^{\star \star} P<0.01\right)$.

and relative proportions (Figure 1) and heat-map and principal component (PCA) analyses (Figure 2), demonstrate that the intestinal microbiota stabilizes at about age 3 years. However, detailed observation of major 11 intestinal bacterial groups (Figure 1A) reveals that, although the numbers of predominant obligate anaerobes stabilize by age 3 years, several LCCM, including Prevotella, Enterobacteriaceae, and Enterococcus, are still in the process of transition have not yet stabilized. Also, as reported in previous studies (Hayashi et al., 2003; Bartosch et al., 2004; Woodmansey et al., 2004; Mitsuoka, 2005; Mueller et al., 2006; Tiihonen et al., 2008; Enck et al., 2009; Mariat et al., 2009), in elderly people the levels of Bifidobacterium are decreased, whereas those of Lactobacillus and Enterobacteriaceae are increased (Figures 1A,B). On the basis of hierarchical clustering of the dataset, we were able to classify the 1951 subjects into four groups, namely AI, AII, BI, and BII (Figure 2A); $94.8 \%$ of subjects belonging to groups AI and AII were aged 3 years or older (adult type), whereas $99.7 \%$ of subjects from groups BI and BII were aged 6 months or less (infant group) (Figure 2B). Notably, these age-related differences are further clearly corroborated by differences in the PCA components of infant-type vs. adult-type cohorts (Figures 2C,D).

Recently, we reported that the counts of predominant obligate anaerobes (C. coccoides group, C. leptum subgroup, $B$. fragilis group and Bifidobacterium) and low-cell-count facultative anaerobes (Enterobacteriaceae, Staphylococcus, and Lactobacillus) follow an approximately logarithmic normal distribution in a cohort of healthy Japanese volunteers aged 3 to 102 years (Nomoto et al., 2015), and this observation prompted us to further analyze and estimate this distribution in relation to host age. We created kernel density estimation plots depicting the distribution of bacterial counts in subjects aged 3 years and older (Figure 3). By using Hartigan's dip test statistics, we found that the distributions of almost all major bacterial groups exhibited a unimodal pattern, which could be considered to be an approximately log-normal distribution. 

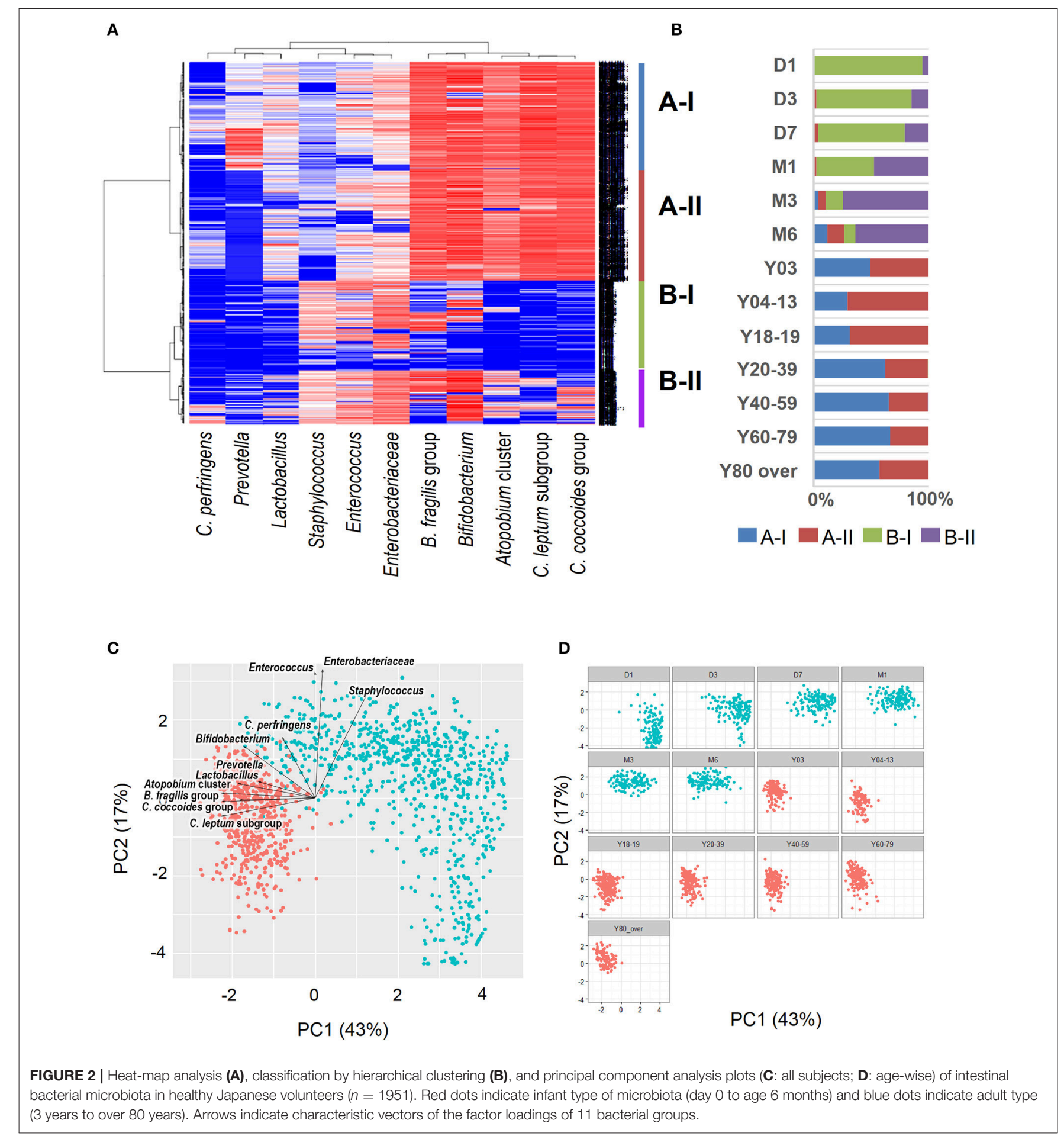

However, Prevotella seems to be an exception: its distribution exhibited a significant multimodality, particularly in subjects aged 18 to 19 and 60 to 79 years (Table 2). Some specific intestinal bacteria (B. fragilis group, Dialister spp., Prevotella group, uncultured Clostridiales UCI, and UCII) have previously been reported to exhibit bimodal abundance distributions and to form stable states at certain abundance levels associated with host factors such as age and obesity (Lahti et al., 2014). This might corroborate our data on the Prevotella distribution in adults. The reason for the multimodal distribution of Prevotella is unclear, but plausible causes are diet, age, sex, and genetic factor differences in adulthood. Alternatively, several Prevotella spp. may exist simply following the rule of multiple stable configurations in the intestine (Gonze et al., 

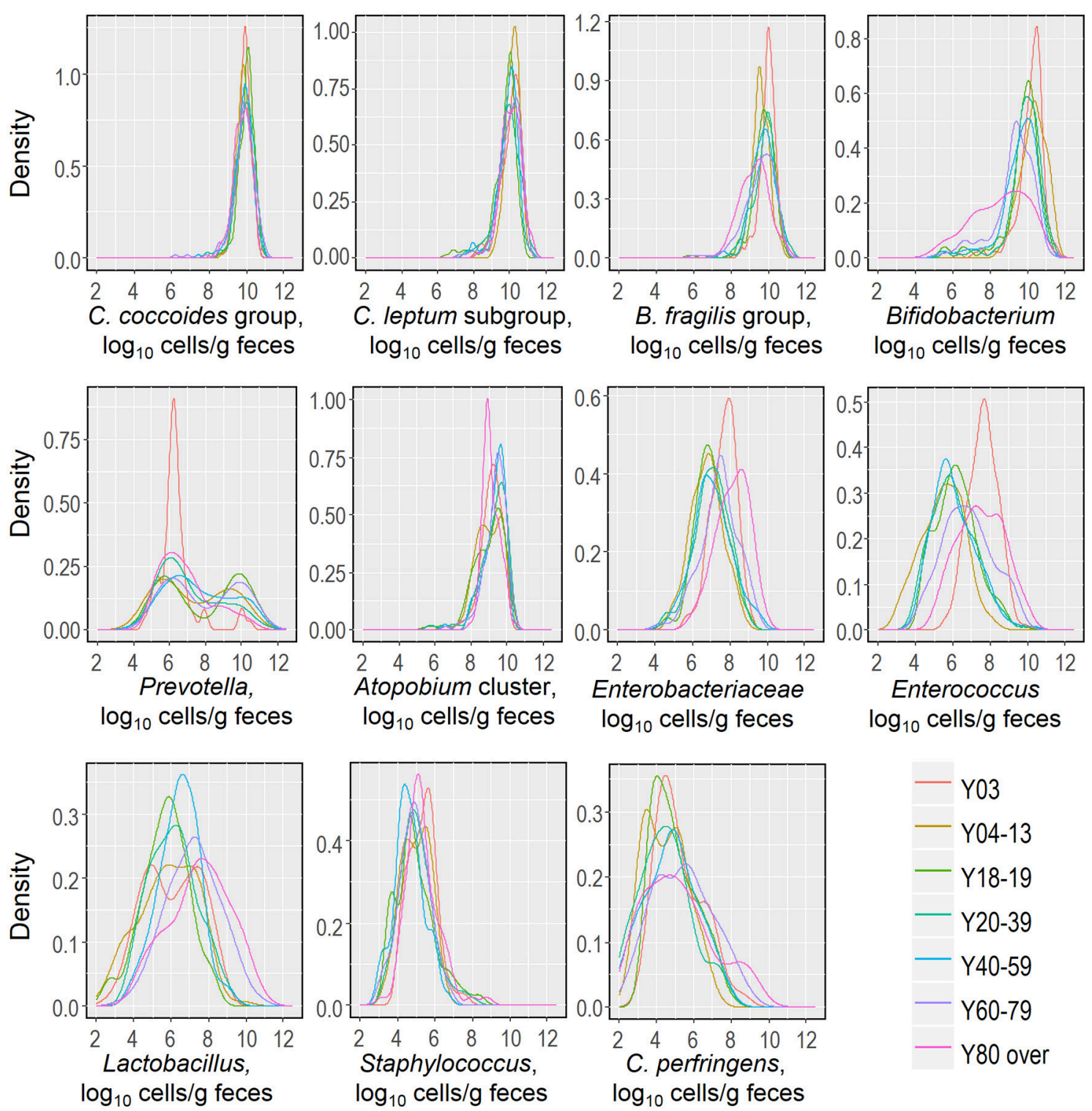

FIGURE 3 | Distributions of fecal counts of major intestinal bacterial groups in healthy Japanese subjects aged 3 years or older $(n=1116)$.

2017). Both Hartigan's dip test (Table 2) and the histogram peaks (Figure 3) clearly demonstrated that all the major gut bacterial clades-except Prevotella-followed an approximately logarithmic normal distribution. Shade et al. (2012) reported that microbial community stability includes resistance and resilience responses to press or pulse disturbances, or both; our result and the above mentioned previous result (Lahti et al., 2014) suggest that the logarithmic normal distribution of bacterial counts indicates that gut bacterial communities are in a state of equilibrium, and that this may be an indicator of a subject's health.

In addition to defining a quantitative view of the core microbiota and the major gut bacterial taxa, YIF-SCAN enables a more detailed analysis of these bacterial groups. For example, in a systematic and quantitative molecular phylogenetic analysis based on the 16S rRNA gene sequence, we classified the $C$. coccoides group (clostridial cluster XIVa $=$ Lachnospiraceae), which consist of many bacterial genera or species with a wide variety of functions, into 19 species and three genetically similar subgroups, and specific primers were newly developed for each (Kurakawa et al., 2015a). Further analysis of these taxa in infants, adults, and elderly subjects revealed that (a) the genus Blautia and the species Ruminococcus gnavus predominated in all age groups, and (b) the genus Dorea and the species Fusicatenibacter saccharivorans were present in high-cell-counts in adults, whereas (c) Clostridium scindens (a secondary bileacid-producing bacterium) prevailed in the elderly. Various factors, such as diet and lifestyle, have an impact on differences 


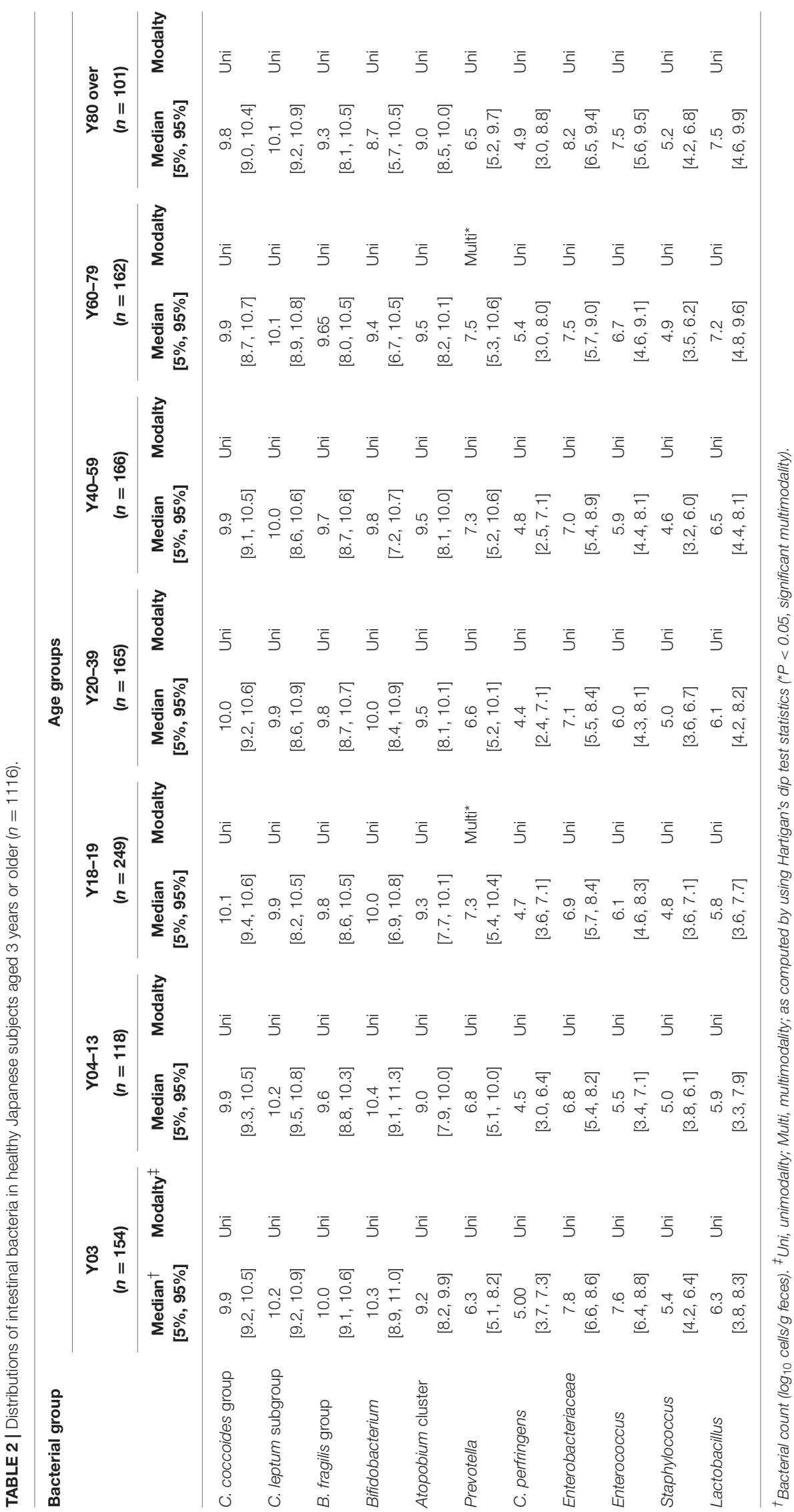


in the predominant anaerobes among age groups (Brown et al., 2012; Clarke et al., 2014; David et al., 2014). Efforts are already under way to further advance the YIF-SCAN system to analyze not only the $C$. coccoides group but also the other predominant groups; we aim to achieve a more comprehensive understanding of the microbiota in the context of factors influencing its composition and functions.

Several recent studies have suggested a link between the gut microbiota and host gender and sex hormones (Mueller et al., 2006; Li et al., 2008; Qin et al., 2010; Bolnick et al., 2014; Shastri et al., 2015; Haro et al., 2016; Oki et al., 2016). Prompted by this concept, we also analyzed our dataset of healthy Japanese young adults according to host gender. Interestingly, we came across several gender-specific differences. For instance, we found that females had higher counts of total bacteria, bifidobacteria, and Lactobacillus gasseri subgroup than their male counterparts (Suzuki et al., 2017). Furthermore, we noted several gender-specific differences in the association of gut microbiota with dietary habits, such as the frequency of yogurt consumption (Suzuki et al., 2017). Although the mechanisms underlying this association remain largely unclear, these genderspecific differences in intestinal bacterial carriage warrant further exploration.

\section{FEATURES OF THE GUT MICROBIOTA OF ASIANS AND OCEANIANS}

Because RNA is more prone to degradation than DNA, the YIF-SCAN system requires the RNA content of samples to be maintained in fresh and RNase-free conditions. This has been achieved by treating the fecal specimen with the commercially available fixing agent RNAlater. By using this method (which has already been validated), the microbial nucleic acids in the homogenized samples can be stably maintained (Kurakawa et al., 2012; Nomoto et al., 2015) under both refrigerated conditions and at ambient temperatures for up to 4 weeks, thereby making it possible to collect and preserve fecal samples, particularly in those settings where samples cannot be preserved at low temperature. This has enabled us to investigate the microbiota of not only Japanese populations but also people from other Asian countries. In one recent study (Nakayama et al., 2015), the gut microbiota of over 300 children from 10 cities in five Asian countries was investigated by simultaneously performing both the exhaustive analysis of $16 \mathrm{~S}$ rRNA genes by pyrosequencing and the quantitative analysis of $16 \mathrm{~S}$ rRNA by YIF-SCAN. The results revealed that the microbiota of these Asian children could be classified into two enterotype-like clusters: a Bacteroides- and Bifidobacteriumrich type and a Prevotella-rich type. Further investigation of the children's dietary and lifestyle factors revealed that the distribution of these two types was correlated with their intake of resistant starch. YIF-SCAN analysis has also revealed unique features of the gut microbiota of people living a subsistence lifestyle in Papua New Guinea (Greenhill et al., 2015). Papua New Guineans consume several starchy crops as their staple foods, and their total protein intake is limited. We can surmise that their microbiota has adapted to these nutritional features.

\section{ANALYZING THE GUT MICROBIOTA AND DETERMINING DYSBIOSIS IN DISEASE STATES}

The highly sensitive YIF-SCAN RT-qPCR detection system has enabled us to easily and precisely detect perturbations in the counts of predominant bacteria as well as LCCM bacteria in patients with diseases such as colorectal cancer, type 2 diabetes, major depressive disorder, anorexia nervosa, Parkinson's disease, inflammatory bowel disease, and ischemic stroke. The counts of the predominant obligate anaerobes and the concentrations of organic acids were significantly lower in colorectal cancer (CRC) patients than in healthy controls (Ohigashi et al., 2013). Among CRC patients with different Duke's stages there were no differences in the microbiota or in organic acid concentrations. Therefore, these changes are not the result of CRC progression but may be related to CRC onset (Ohigashi et al., 2013). YIF-SCAN analysis revealed for the first time the presence of gut dysbiosis and possible blood bacterial translocation, which is defined as the passage of viable indigenous bacteria from the gastrointestinal tract to extraintestinal sites, in Japanese type 2 diabetes (T2D) patients (Sato et al., 2014). Giving probiotic drinks to patients reduced bacterial translocation, and this reduction was accompanied by an improvement in the dysbiosis (Sato et al., 2017). Messaoudi et al. (2011) reported that the administration of probiotics, including Bifidobacterium and Lactobacillus, could contribute to the mental well-being of human subjects with low levels of stress. The results of YIF-SCAN analysis show that reduced counts of Bifidobacterium and Lactobacillus are more common in patients with major depressive disorder (MDD) than in healthy controls (Aizawa et al., 2016). Anorexia nervosa (AN) patients have significantly lower counts of total bacteria and predominant obligate anaerobes than healthy controls (Morita et al., 2015). Moreover, detailed analysis has detected $C$. difficile only in AN binge-eating/purging-subtype patients and not in either healthy subjects or AN restrictive patients (Morita et al., 2015). Cell counts of predominant anaerobic bacteria are significantly lower in Parkinson's disease (PD) patients than in healthy subjects (Hasegawa et al., 2015). A 2-year follow-up study by the same group recently revealed that lower counts of Bifidobacterium and Bacteroides fragilis in year 0 were associated with worsening on the Unified Parkinson's Disease Rating Scale over the next 2 years, suggesting that changes in the intestinal microbiota are associated with the progression of PD (Minato et al., 2017). Counts of $C$. coccoides group bacteria, such as F. saccharivorans, in active ulcerative colitis (UC) patients are lower than those in patients with quiescent UC or in healthy individuals (Takeshita et al., 2016). In a murine acute colitis model, a heat-killed preparation of a $F$. saccharivorans strain isolated from human feces markedly inhibited colonic inflammation (Takeshita et al., 2016). Multivariable linear regression analysis has revealed that ischemic stroke (IS) is independently associated with increased 
bacterial counts of Atopobium cluster and Lactobacillus ruminis subgroup. Furthermore, the changes in the prevalence of $L$. ruminis subgroup are positively correlated with changes in serum interleukin-6 levels, indicating that gut dysbiosis in IS patients is associated with host inflammation (Yamashiro et al., 2017).

These studies collectively demonstrate that the intestinal microbial cell counts of various predominant anaerobes are reduced in patients with mental or neurological disorders such as MDD (Aizawa et al., 2016), PD (Hasegawa et al., 2015), or AN (Morita et al., 2015), as well as in patients with CRC (Ohigashi et al., 2013), T2D (Sato et al., 2014), or IS (Yamashiro et al., 2017) (Table 3). In addition, gut dysbiosis has been reported in patients with inflammatory bowel disease (Takaishi et al., 2008; Carding et al., 2015; Takeshita et al., 2016) or other severe or critical illnesses (Hayakawa et al., 2011), as well as in patients undergoing surgery or chemotherapy (Aoki et al., 2012; Tanaka et al., 2012; Okazaki et al., 2013; Komatsu et al., 2016; Motoori et al., 2017) (Table 3). No doubt, such quantitative elucidations of the commonalities and specificities of dysbiosis in various disorders or during and after surgery are important in that they could help to reveal factors associated with the dysbiosis, facilitate disease diagnosis and prognostication, and improve therapeutic outcomes. Notably, reduced fecal concentrations of organic acids, probably because of reduced levels of predominant anaerobes, have been observed in some diseases (Takaishi et al., 2008; Ohigashi et al., 2013; Sato et al., 2014).

\section{QUANTIFYING THE EFFECTS OF PROBIOTICS ON HUMAN GUT MICROBIAL COMPOSITION}

Probiotics are defined as "live microorganisms which when administered in adequate amounts confer a health benefit on the host" (Reid, Food and Agricultural Organization of the United Nation and WHO, 2005; Hill et al., 2014). In Fuller's (1989) definition, which had been popular long before the introduction of this new definition, the main mechanism of action of probiotics was cited as "improving ... intestinal microbial balance." The current generalized definition fits more appropriately, in particular in the context of the evermounting evidence suggesting that a variety of mechanisms of action, including immune regulation, are at work. However, in essence, the original hypothesis that improving the intestinal microbiota-or, more specifically, normalizing the dysbiosis specific to various pathologic conditions-can effectively prevent or improve pathologies remains very solid. In this context, YIFSCAN-based analysis has enabled us to quantitatively monitor the effects of probiotics on the composition of the gut microbiota. In a study of elderly residents of aged-care facilities, we have shown marked improvement of gut dysbiosis-such as a reduction in the levels of Enterobacteriaceae and indigenous pathobionts and an increase in the numbers of anaerobes usually predominant in the healthy adult gut-following continuous consumption of a probiotic beverage (a fermented milk product containing Lactobacillus casei strain Shirota) for 6 months (Nagata et al., 2016). In another study in healthy children (Wang et al., 2015), we have reported reduced levels of pathobionts (Enterobacteriaceae and Staphylococcus) and increased levels of beneficial bacterial groups (bifidobacteria and lactobacilli) after long-term continuous consumption of probiotics (the same probiotic beverage used in the above-mentioned study of elderly residents). In several other studies, too, we have normalized gut dysbiosis by probiotic administration to different cohorts of gastrectomized subjects and residents of aged-care facilities (Nagata et al., 2011; Aoki et al., 2014). Generally, the levels of indigenous pathobionts such as C. perfringens and Pseudomonas species are extremely low in feces, thereby making it difficult to precisely track their intestinal carriage. To this end, the high sensitivity and precision of RT-qPCR facilitate the analysis of the actual numbers of these pathobionts, thereby also potentially enabling us to control their levels. Indeed, this ability to analyze the intestinal microbiota over a wide dynamic range is very important for elucidating the enhancement of microbiota resilience by probiotics, as well as for identifying potential microbiota-based interventions to ameliorate disease.

\section{APPLICATIONS IN CLINICAL DIAGNOSIS}

From the viewpoint of clinical diagnosis, the precise and sensitive detection and enumeration of disease-causing organisms (which are generally present in low numbers in healthy individuals) becomes indispensable. In a study of patients with febrile neutropenia (Sakaguchi et al., 2010), we compared the use of the blood culture method with our RT-qPCR-based method for diagnosing bacteremia. Whereas the rate of detection of bacteria in the peripheral blood of patients was $17 \%$ with the blood culture method, YIF-SCAN analysis revealed bacteremia in as many as $70 \%$ of patients with fever and neutropenia. Also, in a comparison of the detection of sepsis by using YIF-SCAN and the conventional culture method, YIF-SCAN analysis yielded significantly greater detection rates than the blood culture method (Fujimori et al., 2010). In view of the fact that, for some pediatric patients for whom prompt treatment is required, therapy is sometimes started even before the blood culture results become available, the rapid and highly sensitive analytic ability of the RT-qPCR method could prove particularly useful in a clinical laboratory testing. We have also applied the system in the field of gastrointestinal surgery to investigate bacterial translocation during surgery (Mizuno et al., 2010; Yokoyama et al., 2014). Postoperative infectious complications due to long and complicated surgery, particularly in biliary tract cancer, are a major problem. We have investigated in detail the occurrence of bacterial translocation in patients with biliary tract cancer by examining the mesenteric lymph nodes at the beginning and end of surgery (Mizuno et al., 2010). Intestinal bacteria were detected in $29.4 \%$ of mesenteric lymph nodes at the beginning and $37.3 \%$ following surgery, suggesting that there was a marked increase in bacterial translocation. Further examination of bacterial gene sequences showed that the bacterial strains detected in the blood were the same as those detected in the intestine. In addition, the incidence of 


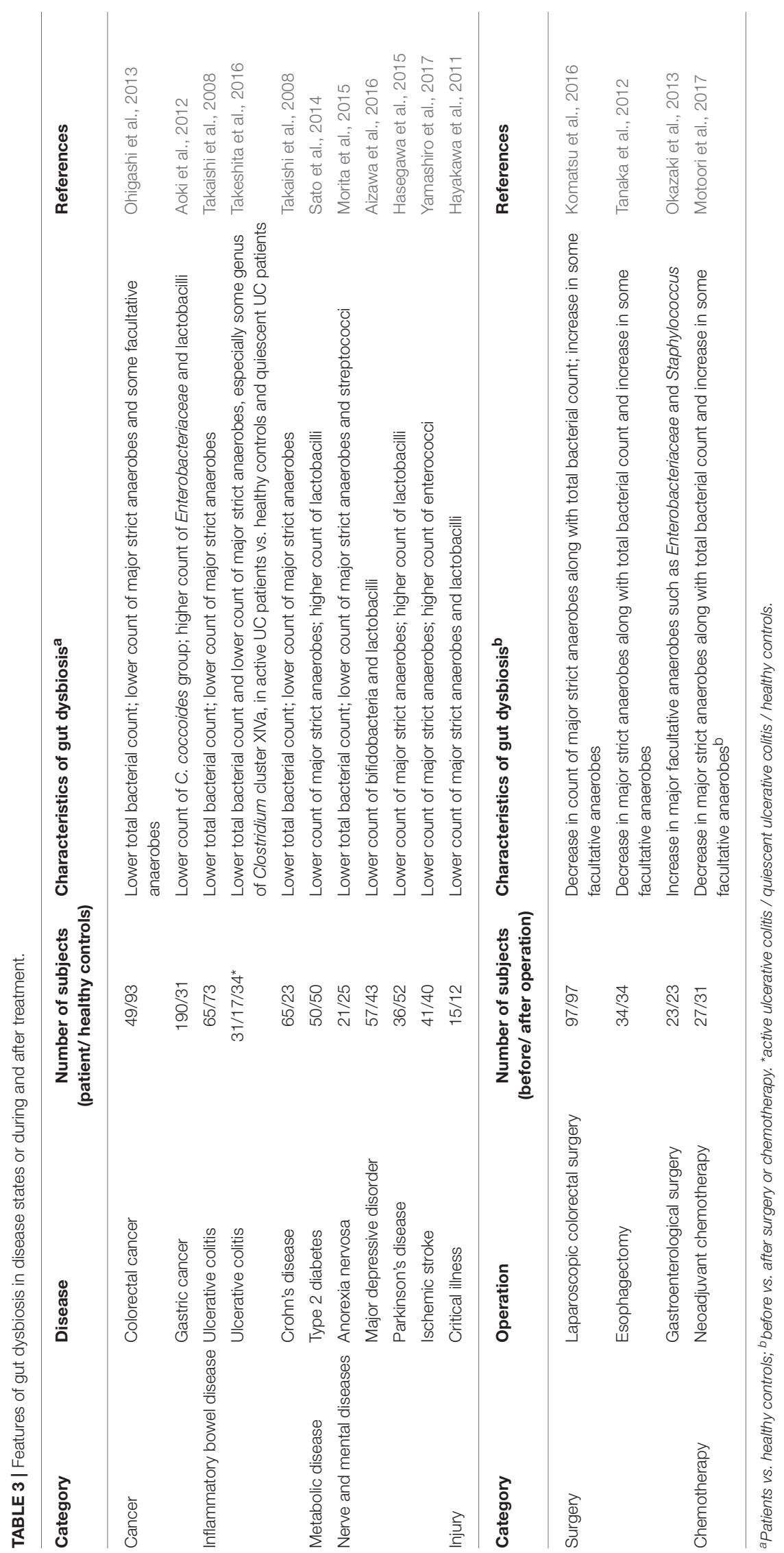


postoperative infectious complications was significantly higher in patients with bacterial translocation. These results clearly suggest that controlling bacterial translocation during surgery can play an important role in preventing postoperative infection. In a subsequent study, we showed that the perioperative use of synbiotics (combinations of probiotics and prebiotics) in patients with esophageal cancer could significantly reduce both bacterial translocation during surgery and the incidence of postoperative infectious complications (Yokoyama et al., 2014).

YIF-SCAN can be used not only for precise analysis of intestinal microbiota but also for sensitive laboratory testing. Culture methods are still typically employed in microbial diagnosis in clinical practice; however, in the case of difficultto-culture and antibiotic-resistant pathogens (e.g., in the case of febrile neutropenia) it is difficult to arrive at a clear-cut diagnosis. In this context, we have been exploring the potential of YIF-SCAN in identifying pathogenic microbes. For example, selection pressure in the targeted culturing of $C$. difficile, the leading cause of antibiotic-associated diarrhea, markedly reduces quantification accuracy. By targeting 23S rRNA molecules, we have established a sensitive and accurate quantification system that can detect $C$. difficile even if its intestinal microbial cell counts are very low (Matsuda et al., 2012). Similarly, to facilitate the diagnosis of Candida, which is a common cause of mycosis and possesses species-specific differences in patterns of resistance to antimicrobial agents, we have validated a highly specific and sensitive quantification system for major pathogenic Candida species, including Candida albicans, Candida glabrata, Candida tropicalis, Candida parapsilosis, and Candida krusei (Ogata et al., 2015). With all of these attributes, YIF-SCAN can facilitate the rapid, efficient, and highly sensitive diagnosis of a wide variety of clinically problematic microbes.

The Human Microbiome Project is analyzing the bacterial gene content of the microbiota in a variety of body niches, including the gastrointestinal tract, oral cavity, skin, and vagina. In the context of the core microbiome concept, the importance of maintaining the stability of the bacterial microbiota of the intestine and vagina has been particularly emphasized (Huse et al., 2012). In this regard, YIF-SCAN has been proven to be an appropriate method for quantifying the various major bacterial groups constituting the vaginal microbiota across a wide range of bacterial numbers. These groups include Lactobacillus subgroups and species, as well as pathobionts such as Gardnerella vaginalis and Atopobium vaginae and pathogens causing sexually transmitted diseases. This YIF-SCAN is based on the analysis of RNA extracted from vaginal mucosal samples and is highly sensitive (Kurakawa et al., 2015b). The system has been used to examine the vaginal bacterial microbiota of healthy Japanese women. In examining the predominance of the Lactobacillus community, we noticed that the counts of several bacterialvaginosis-associated bacteria were quite high in a number of asymptomatic subjects (Kurakawa et al., 2015b) This suggests that there is some sort of early-stage dysbiosis of the vaginal microbiota in the process of bacterial vaginosis. This finding needs to be clarified in further studies, and future research developments could make it possible to maintain a healthy vaginal microbiota as well as to prevent and improve various urogenital diseases by preventing dysbiosis.

\section{CONCLUSION AND PROSPECTS}

This novel approach to bacterial enumeration using rRNAmolecule-targeting RT-qPCR has been remarkably helpful in quantifying the dynamic changes occurring in the gut microbiota during host aging, especially during infancy and early childhood. By using a large integrated database of healthy Japanese people, we have revealed that not only the predominant obligate anaerobes but also facultative anaerobes constitute the core microbiota, and that the counts of each bacterial group approximate a log-normal distribution. We are sure that the information generated from this large integrated cohort will be helpful in further elucidating the features of the gut microbiota in prospective studies. All in all, microbial investigations using YIF-SCAN-based analyses have increased our knowledge of the structure, function, and dynamics of the complex microbial ecosystem both in health and in disease. The ultimate goal is to capitalize on this knowledge to improve human health. We are convinced that expanding the detection range of this analytical system to more microbial clades will provide a more comprehensive picture of the microbiota. Certainly, the scope of this valuable analytical tool will be realized in settings beyond the gastrointestinal tract, and its range of applications will broaden to cover various other niches of the human body and the microbiota of other host species in other biological settings.

\section{AUTHOR CONTRIBUTIONS}

HT analyzed data. HT and KN wrote manuscript. HT, KM, and KN checked and revised manuscript. HT, KM, and KN approved final version of manuscript.

\section{FUNDING}

The study was funded by Yakult Honsha Co. Ltd., Japan. The funding body did not have any additional role in the data analyses, decision to publish, or preparation of the manuscript.

\section{ACKNOWLEDGMENTS}

We thank Norikatsu Yuki, Yukiko Kado, and Kaoru Moriyama (Yakult Central Institute) for their technical advice and help. We also thank Dr. Ravinder Nagpal (Juntendo University School of Medicine) for his cooperation in this study. And, we thank Prof. Colin Hill (University Collage Cork) very much for his English editing of the manuscripts.

\section{SUPPLEMENTARY MATERIAL}

The Supplementary Material for this article can be found online at: https://www.frontiersin.org/articles/10.3389/fmicb. 2018.01417/full\#supplementary-material 


\section{REFERENCES}

Aizawa, E., Tsuji, H., Asahara, T., Takahashi, T., Teraishi, T., Yoshida, S., et al. (2016). Possible association of Bifidobacterium and Lactobacillus in the gut microbiota of patients with major depressive disorder. J. Affect. Disord. 202, 254-257. doi: 10.1016/j.jad.2016.05.038

Aoki, T., Asahara, T., Matsumoto, K., Takada, T., Chonan, O., Nakamori, K., et al. (2014). Effects of the continuous intake of a milk drink containing Lactobacillus casei strain Shirota on abdominal symptoms, fecal microbiota, and metabolites in gastrectomized subjects. Scand. J. Gastroenterol. 49, 552-563. doi: 10.3109/00365521.2013.848469

Aoki, T., Yamaji, I., Hisamoto, T., Sato, M., and Matsuda, T. (2012). Irregular bowel movement in gastrectomized subjects: bowel habits, stool characteristics, fecal flora, and metabolites. Gastric Cancer 15, 396-404. doi: 10.1007/s10120-011-0129-y

Arumugam, M., Raes, J., Pelletier, E., Le Paslier, D., Yamada, T., Mende, D. R., et al. (2011). Enterotypes of the human gut microbiome. Nature 473, 174-180. doi: 10.1038 /nature09944

Bartosch, S., Fite, A., Macfarlane, G. T., and McMurdo, M. E. T. (2004). Characterization of bacterial communities in feces from healthy elderly volunteers and hospitalized elderly patients by using real-time PCR and effects of antibiotic treatment on the fecal microbiota. Appl. Environ. Microbiol. 70, 3575-3581. doi: 10.1128/AEM.70.6.3575-3581.2004

Biagi, E., Nylund, L., Candela, M., Ostan, R., Bucci, L., Pini, E., et al. (2010). Through ageing, and beyond: gut microbiota and inflammatory status in seniors and centenarians. PLoS ONE 5:e10667. doi: 10.1371/journal.pone.0010667

Bian, L., Nagata, S., Asahara, T., Rahman, M. S., Ohta, T., Yuki, N., et al. (2011). Effects of the continuous intake of Lactobacillus casei strain Shirota- fermented milk on risk management of long-term inpatients at health. Int. J. Probiotics Prebiotics 6, 123-132.

Bolnick, D. I., Snowberg, L. K., Hirsch, P. E., Lauber, C. L., Org, E., Parks, B., et al. (2014). Individual diet has sex-dependent effects on vertebrate gut microbiota. Nat. Commun. 5:4500. doi: 10.1038/ncomms5500

Brown, K., DeCoffe, D., Molcan, E., and Gibson, D. L. (2012). Diet-induced dysbiosis of the intestinal microbiota and the effects on immunity and disease. Nutrients 4, 1095-1119. doi: 10.3390/nu4081095

Buffie, C. G., and Pamer, E. G. (2013). Microbiota-mediated colonization resistance against intestinal pathogens. Nat. Rev. Immunol. 13, 790-801. doi: $10.1038 /$ nri3535

Carding, S., Verbeke, K., Vipond, D. T., Corfe, B. M., and Owen, L. J. (2015). Dysbiosis of the gut microbiota in disease. Microb. Ecol. Health Dis. 26:26191. doi: $10.3402 /$ mehd.v26.26191

Cho, I., and Blaser, M. J. (2012). The human microbiome: at the interface of health and disease. Nat. Rev. Genet. 13, 260-270. doi: 10.1038/nrg3182

Claesson, M. J., Jeffery, I. B., Conde, S., Power, S. E., O'Connor, E. M., Cusack, S., et al. (2012). Gut microbiota composition correlates with diet and health in the elderly. Nature 488, 178-184. doi: 10.1038/nature11319

Clarke, S. F., Murphy, E. F., O’Sullivan, O., Lucey, A. J., Humphreys, M., Hogan, A., et al. (2014). Exercise and associated dietary extremes impact on gut microbial diversity. Gut 63, 1913-1920. doi: 10.1136/gutjnl-2013-306541

David, L. A., Maurice, C. F., Carmody, R. N., Gootenberg, D. B., Button, J. E., Wolfe, B. E., et al. (2014). Diet rapidly and reproducibly alters the human gut microbiome. Nature 505, 559-563. doi: 10.1038/nature12820

Enck, P., Zimmermann, K., Rusch, K., Schwiertz, A., Klosterhalfen, S., and Frick, J.-S. (2009). The effects of ageing on the colonic bacterial microflora in adults. Z. Gastroenterol. 47, 653-658. doi: 10.1055/s-0028-1109055

Fujimori, M., Hisata, K., Nagata, S., Matsunaga, N., Komatsu, M., Shoji, H., et al. (2010). Efficacy of bacterial ribosomal RNA-targeted reverse transcriptionquantitative PCR for detecting neonatal sepsis: a case control study. BMC Pediatr. 10:53. doi: 10.1186/1471-2431-10-53

Fuller, R. (1989). Probiotics in man and animals. J. Appl. Bacteriol. 66, 365-378. doi: 10.1111/j.1365-2672.1989.tb05105.x

Gonze, D., Lahti, L., Raes, J., and Faust, K. (2017). Multi-stability and the origin of microbial community types. ISME J. 11, 2159-2166. doi: 10.1038/ismej.2017.60

Gorkiewicz, G. (2009). Nosocomial and antibiotic-associated diarrhoea caused by organisms other than Clostridium difficile. Int. J. Antimicrob. Agents 33(Suppl. 1), S37-S41. doi: 10.1016/S0924-8579(09)70015-9
Greenhill, A. R., Tsuji, H., Ogata, K., Natsuhara, K., Morita, A., Soli, K., et al. (2015). Characterization of the gut microbiota of papua new guineans using reverse transcription quantitative PCR. PLoS ONE 10:e0117427. doi: 10.1371/journal.pone.0117427

Haro, C., Rangel-Zúñiga, O. A., Alcalá-Díaz, J. F., Gómez-Delgado, F., Pérez-Martínez, P., Delgado-Lista, J., et al. (2016). Intestinal microbiota is influenced by gender and body mass index. PLOS ONE 11:e0154090. doi: 10.1371/journal.pone. 0154090

Hasegawa, S., Goto, S., Tsuji, H., Okuno, T., Asahara, T., Nomoto, K., et al. (2015). Intestinal dysbiosis and lowered serum lipopolysaccharidebinding protein in parkinson's disease. PLoS ONE 10:e0142164. doi: 10.1371/journal.pone.0142164

Hayakawa, M., Asahara, T., Henzan, N., Murakami, H., Yamamoto, H., Mukai, N., et al. (2011). Dramatic changes of the gut flora immediately after severe and sudden insults. Dig. Dis. Sci. 56, 2361-2365. doi: 10.1007/s10620-011-1649-3

Hayashi, H., Sakamoto, M., Kitahara, M., and Benno, Y. (2003). Molecular analysis of fecal microbiota in elderly individuals using $16 \mathrm{~S}$ rDNA library and T-RFLP. Microbiol. Immunol. 47, 557-570. doi: 10.1111/j.1348-0421.2003.tb03418.x

Hill, C., Guarner, F., Reid, G., Gibson, G. R., Merenstein, D. J., Pot, B., et al. (2014). Expert consensus document: the international scientific association for probiotics and prebiotics consensus statement on the scope and appropriate use of the term probiotic. Nat. Rev. Gastroenterol. Hepatol. 11, 506-514. doi: $10.1038 /$ nrgastro.2014.66

Hopkins, M. J., Sharp, R., and Macfarlane, G. T. (2001). Age and disease related changes in intestinal bacterial populations assessed by cell culture, $16 \mathrm{~S}$ rRNA abundance, and community cellular fatty acid profiles. Gut 48, 198-205. doi: 10.1136/gut.48.2.198

Huse, S. M., Ye, Y., Zhou, Y., and Fodor, A. A. (2012). A core human microbiome as viewed through $16 \mathrm{~S}$ rRNA sequence clusters. PLoS ONE 7:e34242. doi: 10.1371/journal.pone.0034242

Koeth, R. A., Wang, Z., Levison, B. S., Buffa, J. A., Org, E., Sheehy, B. T., et al. (2013). Intestinal microbiota metabolism of l-carnitine, a nutrient in red meat, promotes atherosclerosis. Nat. Med. 19, 576-585. doi: 10.1038/nm.3145

Komatsu, S., Sakamoto, E., Norimizu, S., Shingu, Y., Asahara, T., Nomoto, K., et al. (2016). Efficacy of perioperative synbiotics treatment for the prevention of surgical site infection after laparoscopic colorectal surgery: a randomized controlled trial. Surg. Today 46, 479-490. doi: 10.1007/s00595-015-1178-3

Kubota, H., Tsuji, H., Matsuda, K., Kurakawa, T., Asahara, T., and Nomoto, K. (2010). Detection of human intestinal catalase-negative, Gram-positive cocci by rRNA-targeted reverse transcription-PCR. Appl. Environ. Microbiol. 76, 5440-5451. doi: 10.1128/AEM.03132-09

Kurakawa, T., Kubota, H., Tsuji, H., Matsuda, K., Asahara, T., Takahashi, T., et al. (2012). Development of a sensitive rRNA-targeted reverse transcription-quantitative polymerase chain reaction for detection of Vibrio cholerae/mimicus, V. parahaemolyticus/alginolyticus and Campylobacter jejuni/coli. Microbiol. Immunol. 56, 10-20. doi: 10.1111/j.1348-0421.2011.00405.x

Kurakawa, T., Kubota, H., Tsuji, H., Matsuda, K., Takahashi, T., Ramamurthy, T., et al. (2013). Intestinal Enterobacteriaceae and Escherichia coli populations in Japanese adults demonstrated by the reverse transcription-quantitative PCR and the clone library analyses. J. Microbiol. Methods 92, 213-219. doi: 10.1016/j.mimet.2012.12.008

Kurakawa, T., Ogata, K., Matsuda, K., Tsuji, H., Kubota, H., Takada, T., et al. (2015a). Diversity of intestinal Clostridium coccoides group in the Japanese population, as demonstrated by reverse transcription-quantitative PCR. PLoS ONE 10:e0126226. doi: 10.1371/journal.pone.0126226

Kurakawa, T., Ogata, K., Tsuji, H., Kado, Y., Takahashi, T., Kida, Y., et al. (2015b). Establishment of a sensitive system for analysis of human vaginal microbiota on the basis of rRNA-targeted reverse transcription-quantitative PCR. J. Microbiol. Methods 111, 93-104. doi: 10.1016/j.mimet.2015. 01.021

Lahti, L., Salojärvi, J., Salonen, A., Scheffer, M., and de Vos, W. M. (2014). Tipping elements in the human intestinal ecosystem. Nat. Commun. 5:4344. doi: $10.1038 /$ ncomms5344

Lakshminarayanan, B., Harris, H. M. B., Coakley, M., O'Sullivan, O., Stanton, C., Pruteanu, M., et al. (2013). Prevalence and characterization of Clostridium perfringens from the faecal microbiota of elderly Irish subjects. J. Med. Microbiol. 62, 457-466. doi: 10.1099/jmm.0.052258-0 
Lawley, T. D., and Walker, A. W. (2013). Intestinal colonization resistance. Immunology 138, 1-11. doi: 10.1111/j.1365-2567.2012.03616.x

Li, M., Wang, B., Zhang, M., Rantalainen, M., Wang, S., Zhou, H., et al. (2008). Symbiotic gut microbes modulate human metabolic phenotypes. Proc. Natl. Acad. Sci. U.S.A. 105, 2117-2122. doi: 10.1073/pnas.0712038105

Litvak, Y., Byndloss, M. X., Tsolis, R. M., and Baumler, A. J. (2017). Dysbiotic proteobacteria expansion: a microbial signature of epithelial dysfunction. Curr. Opin. Microbiol. 39, 1-6. doi: 10.1016/j.mib.2017.07.003

Mariat, D., Firmesse, O., Levenez, F., Guimarăes, V., Sokol, H., Doré, J., et al. (2009). The Firmicutes/Bacteroidetes ratio of the human microbiota changes with age. BMC Microbiol. 9:123. doi: 10.1186/1471-2180-9-123

Matsuda, K., Tsuji, H., Asahara, T., Kado, Y., and Nomoto, K. (2007). Sensitive quantitative detection of commensal bacteria by rRNAtargeted reverse transcription-PCR. Appl. Environ. Microbiol. 73, 32-39. doi: 10.1128/AEM.01224-06

Matsuda, K., Tsuji, H., Asahara, T., Matsumoto, K., Takada, T., and Nomoto, K. (2009). Establishment of an analytical system for the human fecal microbiota, based on reverse transcription-quantitative PCR targeting of multicopy rRNA molecules. Appl. Environ. Microbiol. 75, 1961-1969. doi: 10.1128/AEM.01843-08

Matsuda, K., Tsuji, H., Asahara, T., Takahashi, T., Kubota, H., Nagata, S., et al. (2012). Sensitive quantification of Clostridium difficile cells by reverse transcription-quantitative PCR targeting rRNA molecules. Appl. Environ. Microbiol. 78, 5111-5118. doi: 10.1128/AEM.07990-11

Matsuki, T. (2007). Development of quantitative PCR detection method with $16 \mathrm{~S}$ rRNA gene-targeted genus- and species-specific primers for the analysis of human intestinal microflora and its application. Nippon Saikingaku Zasshi 62, 255-261. doi: $10.3412 /$ jsb.62.255

Matsuki, T., Watanabe, K., Fujimoto, J., Kado, Y., Takada, T., Matsumoto, K., et al. (2004a). Quantitative PCR with $16 \mathrm{~S}$ rRNA-gene-targeted speciesspecific primers for analysis of human intestinal bifidobacteria. Appl. Environ. Microbiol. 70, 167-173. doi: 10.1128/AEM.70.1.167

Matsuki, T., Watanabe, K., Fujimoto, J., Takada, T., and Tanaka, R. (2004b). Use of $16 \mathrm{~S}$ rRNA gene-targeted group-specific primers for real-time PCR analysis of predominant bacteria in human feces. Appl. Environ. Microbiol. 70, 7220-7228. doi: 10.1128/AEM.70.12.7220-7228.2004

Matsuki, T., Watanabe, K., Fujimoto, J., Miyamoto, Y., Takada, T., Matsumoto, K., et al. (2002). Development of $16 \mathrm{~S}$ rRNA-gene-targeted group-specific primers for the detection and identification of predominant bacteria in human feces. Appl. Environ. Microbiol. 68, 5445-5451. doi: 10.1128/AEM.68.11.5445

Matsuki, T., Watanabe, K., Tanaka, R., and Oyaizu, H. (1998). Rapid identification of human intestinal bifidobacteria by $16 \mathrm{~S}$ rRNA-targeted species- and group-specific primers. FEMS Microbiol. Lett. 167, 113-121. doi: 10.1111/j.1574-6968.1998.tb13216.x

Messaoudi, M., Violle, N., Bisson, J.-F. F., Desor, D., Javelot, H., and Rougeot, C. (2011). Beneficial psychological effects of a probiotic formulation (Lactobacillus helveticus R0052 and Bifidobacterium longum R0175) in healthy human volunteers. Gut Microbes 2, 256-261. doi: 10.4161/gmic.2.4.16108

Minato, T., Maeda, T., Fujisawa, Y., Tsuji, H., Nomoto, K., Ohno, K., et al. (2017). Progression of Parkinson's disease is associated with gut dysbiosis: two-year follow-up study. PLoS ONE 12:e0187307. doi: 10.1371/journal.pone.0187307

Mitsuoka, T. (2005). Human microbiota research: present and future. J. Intest. Microbiol 19, 179-192.doi: 10.11209/jim.19.179

Mizuno, T., Yokoyama, Y., Nishio, H., Ebata, T., Sugawara, G., Asahara, T., et al. (2010). Intraoperative bacterial translocation detected by bacteriumspecific ribosomal rna-targeted reverse-transcriptase polymerase chain reaction for the mesenteric lymph node strongly predicts postoperative infectious complications after major hepatectomy for. Ann. Surg. 252, 1013-1019. doi: 10.1097/SLA.0b013e3181f3f355

Morita, C., Tsuji, H., Hata, T., Gondo, M., Takakura, S., Kawai, K., et al. (2015). Gut dysbiosis in patients with anorexia nervosa. PLoS ONE 10:e0145274. doi: 10.1371/journal.pone.0145274

Motoori, M., Yano, M., Miyata, H., Sugimura, K., Saito, T., Omori, T., et al. (2017). Randomized study of the effect of synbiotics during neoadjuvant chemotherapy on adverse events in esophageal cancer patients. Clin. Nutr. 36, 93-99. doi: 10.1016/j.clnu.2015.11.008

Mueller, S., Saunier, K., Hanisch, C., Norin, E., Alm, L., Midtvedt, T., et al. (2006). Differences in fecal microbiota in different European study populations in relation to age, gender, and country: a cross-sectional study. Appl. Environ Microbiol. 72, 1027-1033. doi: 10.1128/AEM.72.2.1027-1033.2006

Nagata, S., Asahara, T., Ohta, T., Yamada, T., Kondo, S., Bian, L., et al. (2011). Effect of the continuous intake of probiotic-fermented milk containing Lactobacillus casei strain Shirota on fever in a mass outbreak of norovirus gastroenteritis and the faecal microflora in a health service facility for the aged. Br. J. Nutr. 106, 549-556. doi: 10.1017/S000711451100064X

Nagata, S., Asahara, T., Wang, C., Suyama, Y., Chonan, O., Takano, K., et al. (2016). The effectiveness of Lactobacillus beverages in controlling infections among the residents of an aged care facility: a randomized placebo-controlled double-blind trial. Ann. Nutr. Metab. 68, 51-59. doi: 10.1159/000442305

Nagpal, R., Ogata, K., Tsuji, H., Matsuda, K., Takahashi, T., Nomoto, K., et al. (2015). Sensitive quantification of Clostridium perfringens in human feces by quantitative real-time PCR targeting alpha-toxin and enterotoxin genes. $B M C$ Microbiol. 15:219. doi: 10.1186/s12866-015-0561-y

Nair, G. B., Ramamurthy, T., Sur, D., Kurakawa, T., Takahashi, T., Nomoto, K., et al. (2012). Vibrio cholerae/mimicus in fecal microbiota of healthy children in a cholera endemic urban slum setting in Kolkata, India. Microbiol. Immunol. 56, 789-791. doi: 10.1111/j.1348-0421.2012.00497.x

Nakayama, J. (2010). Pyrosequence-based 16S rRNA profiling of gastro-intestinal microbiota. Biosci. Microflora 29, 83-96. doi: 10.12938/bifidus.29.83

Nakayama, J., Watanabe, K., Jiang, J., Matsuda, K., Chao, S.-H., Haryono, P., et al. (2015). Diversity in gut bacterial community of school-age children in Asia. Sci. Rep. 5:8397. doi: 10.1038/srep08397

Nomoto, K., Tsuji, H., and Matsuda, K. (2015). YIF-SCAN ${ }^{\circledR}$ : a novel system for intestinal microflora analysis. J. Intest. Microbiol. 29, 9-18. doi: $10.11209 /$ jim. 29.9

O'Toole, P. W. (2012). Changes in the intestinal microbiota from adulthood through to old age. Clin. Microbiol. Infect. 18(Suppl. 4), 44-46. doi: 10.1111/j.1469-0691.2012.03867.x

Odamaki, T., Kato, K., Sugahara, H., Hashikura, N., Takahashi, S., Xiao, J., et al. (2016). Age-related changes in gut microbiota composition from newborn to centenarian: a cross-sectional study. BMC Microbiol. 16:90. doi: 10.1186/s12866-016-0708-5

Ogata, K., Matsuda, K., Tsuji, H., and Nomoto, K. (2015). Sensitive and rapid RT-qPCR quantification of pathogenic Candida species in human blood. J. Microbiol. Methods 117, 128-135. doi: 10.1016/j.mimet.2015.07.021

Ohigashi, S., Sudo, K., Kobayashi, D., Takahashi, O., Takahashi, T., Asahara, T., et al. (2013). Changes of the intestinal microbiota, short chain fatty acids, and fecal pH in patients with colorectal cancer. Dig. Dis. Sci. 58, 1717-1726. doi: 10.1007/s10620-012-2526-4

Okazaki, M., Matsukuma, S., Suto, R., Miyazaki, K., Hidaka, M., Matsuo, M., et al. (2013). Perioperative synbiotic therapy in elderly patients undergoing gastroenterological surgery: a prospective, randomized control trial. Nutrition 29, 1224-1230. doi: 10.1016/j.nut.2013.03.015

Oki, K., Toyama, M., Banno, T., Chonan, O., Benno, Y., and Watanabe, K. (2016). Comprehensive analysis of the fecal microbiota of healthy Japanese adults reveals a new bacterial lineage associated with a phenotype characterized by a high frequency of bowel movements and a lean body type. BMC Microbiol. 16:284. doi: 10.1186/s12866-016-0898-x

Ozaki, E., Kato, H., Kita, H., Karasawa, T., Maegawa, T., Koino, Y., et al. (2004). Clostridium difficile colonization in healthy adults: transient colonization and correlation with enterococcal colonization. J. Med. Microbiol. 53, 167-172. doi: 10.1099/jmm.0.05376-0

Qin, J., Li, R., Raes, J., Arumugam, M., Burgdorf, K. S., Manichanh, C., et al. (2010). A human gut microbial gene catalogue established by metagenomic sequencing. Nature 464, 59-65. doi: 10.1038/nature08821

Rampelli, S., Candela, M., Turroni, S., Biagi, E., Collino, S., Franceschi, C., et al. (2013). Functional metagenomic profiling of intestinal microbiome in extreme ageing. Aging 5, 902-912. doi: 10.18632/aging.100623

Reid, G., and Food and Agricultural Organization of the United Nation and WHO (2005). The importance of guidelines in the development and application of probiotics. Curr. Pharm. Des. 11, 11-16. doi: 10.2174/1381612053382395

Round, J. L., and Mazmanian, S. K. (2009). The gut microbiota shapes intestinal immune responses during health and disease. Nat. Rev. Immunol. 9, 313-323. doi: $10.1038 /$ nri2515

Sakaguchi, S., Saito, M., Tsuji, H., Asahara, T., Takata, O., Fujimura, J., et al. (2010). Bacterial rRNA-targeted reverse transcription-PCR used to identify pathogens 
responsible for fever with neutropenia. J. Clin. Microbiol. 48, 1624-1628. doi: 10.1128/JCM.01724-09

Samarkos, M., Mastrogianni, E., and Kampouropoulou, O. (2018). The role of gut microbiota in Clostridium difficile infection. Eur. J. Intern. Med. 50, 28-32. doi: 10.1016/j.ejim.2018.02.006

Sato, J., Kanazawa, A., Azuma, K., Ikeda, F., Goto, H., Komiya, K., et al. (2017). Probiotic reduces bacterial translocation in type 2 diabetes mellitus: a randomised controlled study. Sci. Rep. 7:12115. doi: 10.1038/s41598-017-12535-9

Sato, J., Kanazawa, A., Ikeda, F., Yoshihara, T., Goto, H., Abe, H., et al. (2014). Gut dysbiosis and detection of "live gut bacteria" in blood of Japanese patients with type 2 diabetes. Diabetes Care 37, 2343-2350. doi: 10.2337/dc13-2817

Shade, A., Peter, H., Allison, S. D., Baho, D. L., Berga, M., Bürgmann, H., et al. (2012). Fundamentals of microbial community resistance and resilience. Front. Microbiol. 3:417. doi: 10.3389/fmicb.2012.00417

Shastri, P., McCarville, J., Kalmokoff, M., Brooks, S. P. J., and Green-Johnson, J. M. (2015). Sex differences in gut fermentation and immune parameters in rats fed an oligofructose-supplemented diet. Biol. Sex Differ. 6:13. doi: 10.1186/s13293-015-0031-0

Suzuki, Y., Ikeda, K., Sakuma, K., Kawai, S., Sawaki, K., Asahara, T., et al. (2017). Association between yogurt consumption and intestinal microbiota in healthy young adults differs by host gender. Front. Microbiol. 8:847. doi: 10.3389/fmicb.2017.00847

Takaishi, H., Matsuki, T., Nakazawa, A., Takada, T., Kado, S., Asahara, T., et al. (2008). Imbalance in intestinal microflora constitution could be involved in the pathogenesis of inflammatory bowel disease. Int. J. Med. Microbiol. 298, 463-472. doi: 10.1016/j.ijmm.2007.07.016

Takeshita, K., Mizuno, S., Mikami, Y., Sujino, T., Saigusa, K., Matsuoka, K., et al. (2016). A single species of Clostridium subcluster XIVa decreased in ulcerative colitis patients. Inflamm. Bowel Dis. 22, 2802-2810. doi: 10.1097/MIB.0000000000000972

Tanaka, K., Yano, M., Motoori, M., Kishi, K., Miyashiro, I., Ohue, M., et al. (2012). Impact of perioperative administration of synbiotics in patients with esophageal cancer undergoing esophagectomy: a prospective randomized controlled trial. Surgery 152, 832-842. doi: 10.1016/j.surg.2012.02.021

Tannock, G. W., Lee, P. S., Wong, K. H., and Lawley, B. (2016). Why don't all infants have bifidobacteria in their stool? Front. Microbiol. 7:834. doi: $10.3389 /$ fmicb. 2016.00834

Tiihonen, K., Ouwehand, A. C., and Rautonen, N. (2010). Human intestinal microbiota and healthy ageing. Ageing Res. Rev. 9, 107-116. doi: 10.1016/j.arr.2009.10.004

Tiihonen, K., Tynkkynen, S., Ouwehand, A., Ahlroos, T., and Rautonen, N. (2008). The effect of ageing with and without non-steroidal anti-inflammatory drugs on gastrointestinal microbiology and immunology. Br. J. Nutr. 100, 130-137. doi: 10.1017/S000711450888871X

Tonooka, T., Sakata, S., Kitahara, M., Hanai, M., Ishizeki, S., Takada, M., et al. (2005). Detection and quantification of four species of the genus Clostridium in infant feces. Microbiol. Immunol. 49, 987-992. doi: 10.1111/j.1348-0421.2005.tb03694.x

Tsuji, H., Chonan, O., Suyama, Y., Kado, Y., Nomoto, K., Nanno, M., et al. (2014). Maintenance of healthy intestinal microbiota in women who regularly consume probiotics. Int. J. Probiotics Prebiotics 9, 31-38.
Tsuji, H., Oozeer, R., Matsuda, K., Matsuki, T., Ohta, T., Nomoto, K., et al. (2012). Molecular monitoring of the development of intestinal microbiota in Japanese infants. Benef. Microbes 3, 113-125. doi: 10.3920/BM2011.0038

Turnbaugh, P. J., Ley, R. E., Hamady, M., Fraser-Liggett, C. M., Knight, R., and Gordon, J. I. (2007). The human microbiome project. Nature 449, 804-810. doi: 10.1038/nature06244

Turnbaugh, P. J., Ley, R. E., Mahowald, M. A., Magrini, V., Mardis, E. R., and Gordon, J. I. (2006). An obesity-associated gut microbiome with increased capacity for energy harvest. Nature 444, 1027-1031. doi: 10.1038/nature05414

Vandeputte, D., Kathagen, G., D’hoe, K., Vieira-Silva, S., Valles-Colomer, M., Sabino, J., et al. (2017). Quantitative microbiome profiling links gut community variation to microbial load. Nature 551, 507-511. doi: 10.1038/nature24460

van der Waaij, D., Berghuis-de Vries, J. M., and Lekkerkerk-van der Wees, J. (1971). Colonization resistance of the digestive tract in conventional and antibiotic-treated mice. J. Hyg. 69, 405-411. doi: 10.1017/S0022172400021653

Wang, C., Nagata, S., Asahara, T., Yuki, N., Matsuda, K., Tsuji, H., et al. (2015) Intestinal microbiota profiles of healthy pre-school and school-age children and effects of probiotic supplementation. Ann. Nutr. Metab. 67, 257-266. doi: $10.1159 / 000441066$

Woodmansey, E. J. (2007). Intestinal bacteria and ageing. J. Appl. Microbiol. 102, 1178-1186. doi: 10.1111/j.1365-2672.2007.03400.x

Woodmansey, E. J., McMurdo, M. E. T., Macfarlane, G. T., and Macfarlane, S. (2004). Comparison of compositions and metabolic activities of fecal microbiotas in young adults and in antibiotic-treated and nonantibiotic-treated elderly subjects. Appl. Environ. Microbiol. 70, 6113-6122. doi: 10.1128/AEM.70.10.6113-6122.2004

Yamashiro, K., Tanaka, R., Urabe, T., Ueno, Y., Yamashiro, Y., Nomoto, K., et al. (2017). Gut dysbiosis is associated with metabolism and systemic inflammation in patients with ischemic stroke. PLOS ONE 12:e0171521. doi: 10.1371/journal.pone.0171521

Yatsunenko, T., Rey, F. E., Manary, M. J., Trehan, I., Dominguez-Bello, M. G., Contreras, M., et al. (2012). Human gut microbiome viewed across age and geography. Nature 486, 222-227. doi: 10.1038/nature11053

Yokoyama, Y., Nishigaki, E., Abe, T., Fukaya, M., Asahara, T., Nomoto, K., et al. (2014). Randomized clinical trial of the effect of perioperative synbiotics versus no synbiotics on bacterial translocation after oesophagectomy. Br. J. Surg. 101, 189-199. doi: 10.1002/bjs.9385

Yoshimoto, S., Loo, T. M., Atarashi, K., Kanda, H., Sato, S., Oyadomari, S., et al. (2013). Obesity-induced gut microbial metabolite promotes liver cancer through senescence secretome. Nature 499, 97-101. doi: 10.1038/nature12347

Conflict of Interest Statement: The authors declare that the research was conducted in the absence of any commercial or financial relationships that could be construed as a potential conflict of interest.

Copyright (c) 2018 Tsuji, Matsuda and Nomoto. This is an open-access article distributed under the terms of the Creative Commons Attribution License (CC $B Y)$. The use, distribution or reproduction in other forums is permitted, provided the original author(s) and the copyright owner are credited and that the original publication in this journal is cited, in accordance with accepted academic practice. No use, distribution or reproduction is permitted which does not comply with these terms. 